\title{
Design of D-band Double Corrugated Waveguide TWT for Wireless Communications
}

\author{
Rupa Basu. Laxma R. Billa, Jeevan M. Rao, Rosa Letizia, Claudio Paoloni \\ Engineering Department \\ Lancaster University \\ Lancaster, UK \\ c.paoloni@lancaster.ac.uk
}

\begin{abstract}
The European Commission Horizon 2020
ULTRAWAVE, "Ultra capacity wireless layer beyond $100 \mathrm{GHz}$ based on millimeter wave Traveling Wave Tubes", aims to exploit portions of two frequency bands in the millimetre wave spectrum, the D-band (141 - 148.5 GHz) and the G-band (275 $305 \mathrm{GHz}$ ) for creating a very high capacity layer. Due to the high atmosphere and rain attenuation, high transmission power is needed to provide a useful transmission range. Traveling Wave Tubes are the only devices that can provide the multi-Watt transmission power above $100 \mathrm{GHz}$. In this paper, the design of the Double Corrugated Waveguide (DCW), as slow wave structure, for a novel D-band TWT, for wireless communications, will be described.
\end{abstract}

Keywords - Millimeter wave networks, traveling wave tube, Double corrugated waveguide, $5 \mathrm{G}$

\section{INTRODUCTION}

The European Union's Horizon 2020 ULTRAWAVE project, "Ultra Capacity Wireless Layer Beyond $100 \mathrm{GHz}$ Based on Millimeter Wave Traveling Wave Tubes", aims to exploit two main frequency bands in the millimetre wave spectrum, the D-band $(141-148.5 \mathrm{GHz})$ and the G-band $(275$ $-305 \mathrm{GHz})[1,2]$, able to support Gigabit per second class data rate. So far, the lack of sufficient transmission power and consequently adequate signal to noise ratio have prevented a useful outdoor range, of a few hundreds of meters, transmission above $100 \mathrm{GHz}$ [2]. ULTRAWAVE project is based on the use of novel millimetre wave traveling wave tubes, that are the presently the only devices able to deliver power at Watt level above $100 \mathrm{GHz}$. Only a few TWTs based on folded waveguide have been demonstrated at prototype level $[3,4]$. In this paper, the design of the D-band (141 $148.5 \mathrm{GHz}$ ) TWT will be described.

In perspective, the deployment of the wireless system will cover wide areas, with the deployment of a large number of front end powered by TWTs. One of the targets of the design approach for the proposed TWT is a low cost with adequate performance. This will permit to build affordable front end suitable for the wireless communication market.

\section{TWT DESIGN}

\section{A. Double Corrugated Waveguide design}

A double corrugated waveguide (DCW) slow wave structure (SWS) was designed to support a beam voltage of 13 $\mathrm{kV}$ [7]. The dimensions were optimised to assure the proper synchronism over the operating frequency band. Fig.1 shows the dispersion curve computed by CST - Eigenmode solver. The wide bandwidth of about $20 \mathrm{GHz}$, satisfies the specifications. An interaction impedance of about $1.5 \Omega$ has been obtained.

\section{B. Sensitivity analysis}

One of the major challenges in fabrication of parts for TWT operating above $100 \mathrm{GHz}$ is the control of fabrication tolerance and to assure a level of accuracy suitable for the required performance. This is particularly important for the electron gun and the slow wave structure (SWS). A SWS that allows a low-cost fabrication and easy assembly would reduce the overall total cost of the TWT [5]. In case of the DCW, a sensitivity analysis was performed to assess the level of accuracy needed by a fabrication process.

Figure 2 shows the variation of the dispersion and the interaction impedance as a function of a variation of $+/-10 \mu \mathrm{m}$ of the period length with respect to the nominal value $(575 \mu \mathrm{m})$. It is notable that a variation of 10 micron determines a variation of the dispersion corresponding to a beam voltage variation of about $200 \mathrm{~V}$. This value can be considered not significant. The operation band and the interaction impedance are not substantially affected. This study was performed for all the critical dimensions to define the maximum tolerance for achieving the performance. A fabrication tolerance of 5 microns has been found very satisfactory for the specific frequency range.

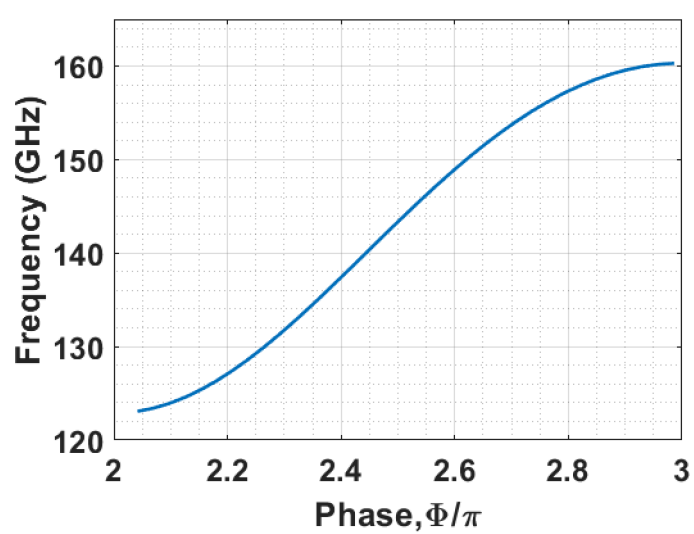

Fig. 1. Double corrugated waveguide dispersion curve

\section{S-parameters}

The second design step was the simulation of the Sparameters of a complete DCW slow wave structure including the input and output couplers. Figure 3 reports the behaviour over the D-band. Very good $\mathrm{S}_{11}$ and the relatively low losses were obtained. 

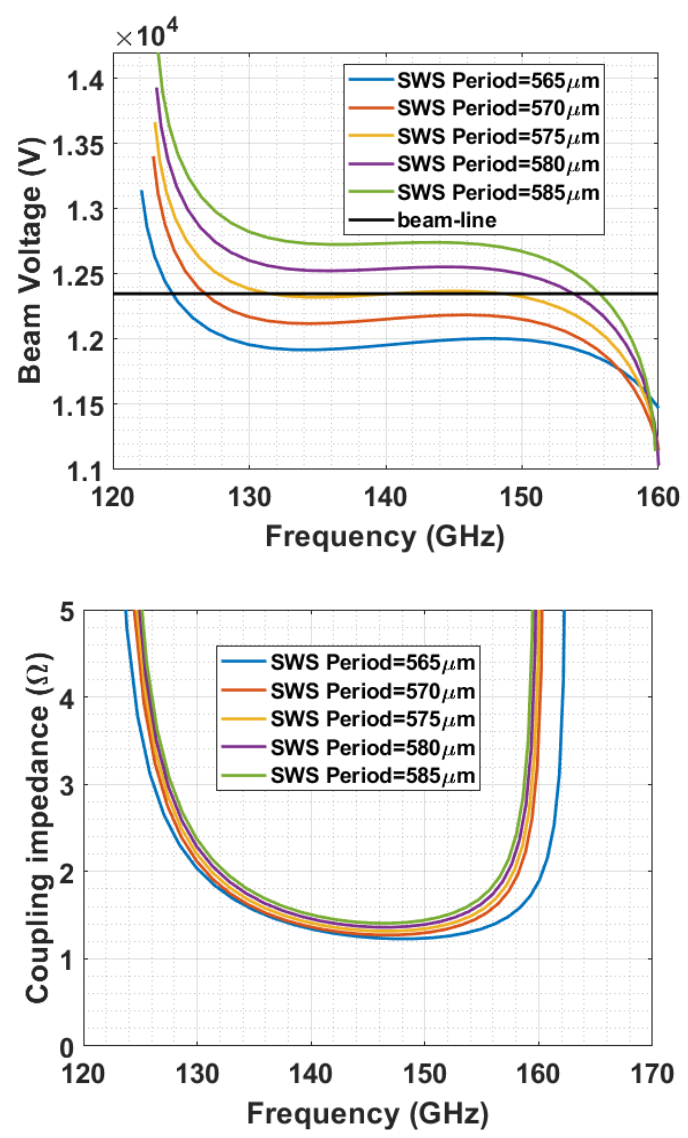

Fig. 2. Sensitivity analysis of dispersion and interaction impedance as a function of the period variation.

\section{Large signal analysis}

The TWT is designed with a two-section DCW separated by a sever, to get about $40 \mathrm{~dB}$ gain. The initial Particle in Cell (PIC) simulations were focussed to a single section, with a limited number of periods, to find the optimum beam voltage and reduce the simulation time. Once the PIC simulations provided good values for power and gain, a full two-section DCW was simulated to achieve the specifications. A sever has been inserted between the two sections to prevent oscillations. The first section is 60 periods long, the second section 110 periods. About $35 \mathrm{~dB}$ gain and $12 \mathrm{~W}$ output power were achieved with $4 \mathrm{~mW}$ input power (Fig. 4). This performance satisfies the specification for the link budget.

\section{CONCLUSIONS}

The design and performance of a two section D-band TWT have been discussed. The Double Corrugated Waveguide was designed to provide output power suitable for long wireless communication links. The simple structure of the DCW will assure a relatively low cost TWT. The fabrication of the TWT is in progress.

\section{ACKNOWLEDGMENT}

The ULTRAWAVE project has received funding from the European Union's Horizon 2020 research and innovation program under grant agreement no 762119.

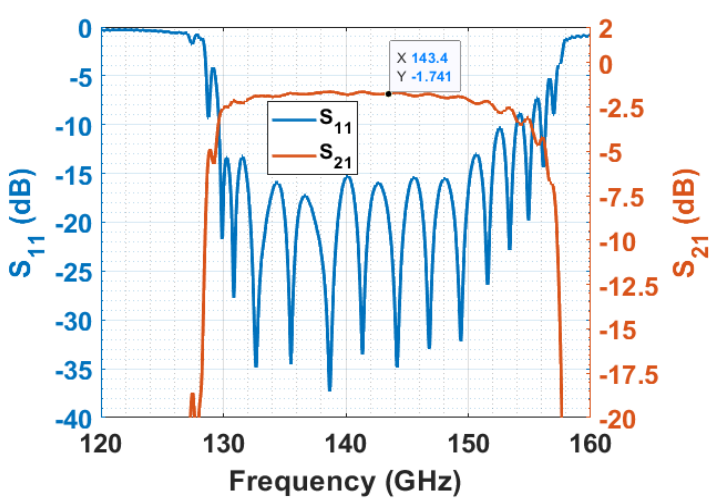

Fig. 3. S-parameters of a single section DCW with 20 periods.

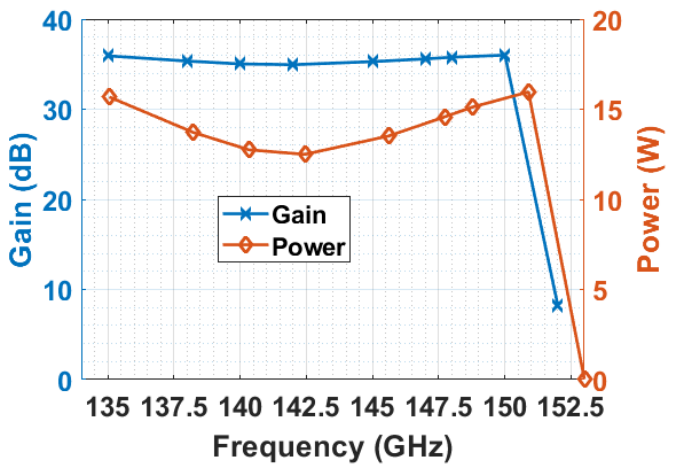

Fig. 4. Gain and output power of two-section DCW TWT

\section{REFERENCES}

[1] http://www.ultrawave2020.eu

[2] Dhillon, S. S., M. S. Vitiello, E. H. Linfield, A. G. Davies, Matthias C. Hoffmann, John Booske, Claudio Paoloni et al. "The 2017 terahertz science and technology roadmap." Journal of Physics D: Applied Physics 50, no. 4 (2017): 043001.

[3] L. Wenqiang et al., "Development of D-band continuous-wave folded waveguide traveling-wave tube," 2015 IEEE International Vacuum Electronics Conference (IVEC), Beijing, 2015

[4] M. Field et al., "Development of a 100-W 200-GHz High Bandwidth mm-Wave Amplifier," in IEEE Transactions on Electron Devices, vol. 65, no. 6, pp. 2122-2128, June 2018.

[5] Joye, Colin D., Jeffrey P. Calame, Khanh T. Nguyen, and Morag Garven. "Microfabrication of fine electron beam tunnels using UVLIGA and embedded polymer monofilaments for vacuum electron devices." Journal of Micromechanics and Microengineering 22, no. 1 (2011): 015010.

[6] Basu, Rupa, Laxma R. Billa, Rosa Letizia, and Claudio Paoloni. "Design of sub-THz traveling wave tubes for high data rate long range wireless links." Semiconductor Science and Technology 33, no. 12 (2018): 124009.

[7] M. Mineo and C. Paoloni, "Double-Corrugated Rectangular Waveguide Slow-Wave Structure for Terahertz Vacuum Devices," IEEE Transactions on Electron Devices, vol. 57, no. 11, pp. 31693175, Nov. 2010 